\title{
Acute transient freezing of gait in a patient with posterior reversible encephalopathy syndrome
}

\author{
Asuka Nakajima', Yuji Ueno ${ }^{1 *}$, Hideki Shimura', Taiki Kambe¹, Kenya Nishioka', Nobutaka Hattori \\ and Takao Urabe ${ }^{?}$
}

\begin{abstract}
Background: Posterior reversible encephalopathy syndrome (PRES) is a transient clinical and neuroradiologic syndrome caused by cerebral vasogenic edema. Various reversible neurologic symptoms were shown in patients with PRES. Freezing of gait (FOG) is mainly observed in neurodegenerative diseases.

Case presentation: We report a 43-year-old man, with undergoing hemodialysis therapy for chronic renal failure, had mild elevation of blood pressure. His consciousness level suddenly deteriorated, and brain MRI demonstrated hyperintense lesions in the bilateral basal ganglia on fluid-attenuated inversion recovery images, diffusion-weighted images, and apparent diffusion coefficient maps. After improvement of disturbance of consciousness, he showed FOG accompanied by bradykinesia and postural instability. His FOG spontaneously improved concurrently with alleviation of basal ganglionic lesions on follow-up MRI.
\end{abstract}

Conclusions: It is suggested that vasogenic edema on bilateral basal ganglia associated with PRES can cause acute transient FOG.

Keywords: Posterior reversible encephalopathy syndrome, Freezing of gait, Parkinsonism, Vasogenic edema, Hemodialysis

\section{Background}

Posterior reversible encephalopathy syndrome (PRES) is usually described as presenting with a variety of reversible neurologic symptoms including headache, seizures, vision loss, and deterioration of consciousness [1,2]. Vasogenic edema caused by a breakdown of autoregulation of cerebral blood flow is proposed as a principal pathogenesis of PRES [1,2]. For the diagnosis of PRES, increasing signal intensity on apparent diffusion coefficient maps (ADC maps) are indispensable to differentiate vasogenic edema from cytotoxic edema, although diffusion-weighted images (DWI) and fluidattenuated inversion recovery (FLAR) images might show high signals in both vasogenic and cytotoxic edemas.

Freezing of gait (FOG), often accompanied by bradykinesia, rigidity, and postural instability, is observed in

\footnotetext{
* Correspondence: yuji-u@juntendo.ac.jp

'Department of Neurology, Juntendo University Urayasu Hospital, 2-1-1

Tomioka, Urayasu, Chiba 279-0021, Japan

Full list of author information is available at the end of the article
}

several neurological disorders including Parkinson's disease and parkinsonian syndromes [3,4].

Here, we report a 43-year-old man who suddenly developed FOG associated with vasogenic edema in the bilateral basal ganglia caused by PRES. His FOG spontaneously improved concurrently with alleviation of basal ganglionic lesions. It is suggested that vasogenic edema on bilateral basal ganglia associated with PRES can cause transient FOG.

\section{Case presentation}

A 43-year-old man, who had undergone hemodialysis therapy for chronic renal failure owing to diabetic nephropathy for 6 years and whose blood pressure had been under control $(<140 / 80 \mathrm{mmHg})$, developed mild elevation of blood pressure $(150 / 100 \mathrm{mmHg})$ for 2 weeks. He has not been treated with immunosuppressant agents. He suddenly showed weakness in both of his legs, and he realized that he had postural instability and that he could not walk. Subsequently, his consciousness level deteriorated. On admission, his blood pressure was $156 / 96 \mathrm{mmHg}$. Neurological 
examination showed a Glasgow Coma Scale of 14 (E3V5M6). His facial expression was flat and his voice was barely audible. He had paraparesis in both lower limbs. Brain MRI demonstrated hyperintense lesions in the bilateral basal ganglia, bilateral frontal cortices, and left cerebellum on FLAIR images, and those lesions were hyperintensity on DWI and ADC maps (Figure 1A-C). No arterial stenosis on MR angiography was found (Figure 1D). He was treated with anti-hypertensive agents and continued hemodialysis therapy, and his blood pressure were controlled to $130 / 70 \mathrm{mmHg}$ within 2 weeks. On the 7 th day after admission, disturbance of consciousness and paraparesis improved, and he could walk by himself. However, we noticed that he showed parkinsonian signs including FOG, bradykinesia, and postural instability. His FOG fulfilled the criteria of "brief, episodic absence or marked reduction of forward progression of the feet despite the intention to walk" based on the 2010 workshop of clinicians and scientists interested in FOG [5]. The score from part III of the Unified Parkinson's Disease Rating Scale (UPDRS) was 28 points. He did not show cerebellar ataxia. Concurrently, MRI showed hyperintense basal ganglionic lesions on FLAIR images, DWI, and ADC maps persisted, although lesions on bilateral frontal cortices were diminished (Figure 2A-C). His FOG and postural instability gradually improved, and UPDRS part III scores were 22 and 5 points at 2 and 4 weeks after admission, respectively. Follow-up MRI at 4 weeks after admission showed significant alleviation of the bilateral basal ganglionic lesions on FLAIR (Figure 2D). Thus, he was diagnosed as PRES based on the acute transient neurological symptoms and MRI findings. He was treated with $150 \mathrm{mg}$ of amantadine, and a combination of $300 \mathrm{mg}$ of levodopa and $30 \mathrm{mg}$ of carbidopa at 15th and 22st day after admission for 7 days, respectively, but he did not show dramatic improvement of FOG. Thereafter, those anti-parkinsonian drugs were discontinued.

\section{Discussion}

The basal ganglia is involved in $12 \%$ to $34 \%$ of patients with PRES, and is especially common in patients with preeclampsia-eclampsia [1,2]. Compared to the cortical gray matter, the basal ganglia contains a large number of non-anastomotic vessels and capillary beds, and may therefore be more vulnerable to increased systemic blood pressure and circulating toxins [6]. While hemodialysis is generally used for the treatment of renal disease, hemodialysis itself may cause vascular endothelial damage [7]. Thus, we concluded that endothelial damage caused by elevation of blood pressure and hemodialysis might be closely associated with vasogenic edema in the bilateral basal ganglia, which resulted in PRES in our case. To the best of our knowledge, we firstly reported that vasogenic edema on bilateral basal ganglia associate with PRES could cause acute parkinsonian freezing gait, and that alleviation of basal ganglionic lesions was consistent with an improvement of his FOG.
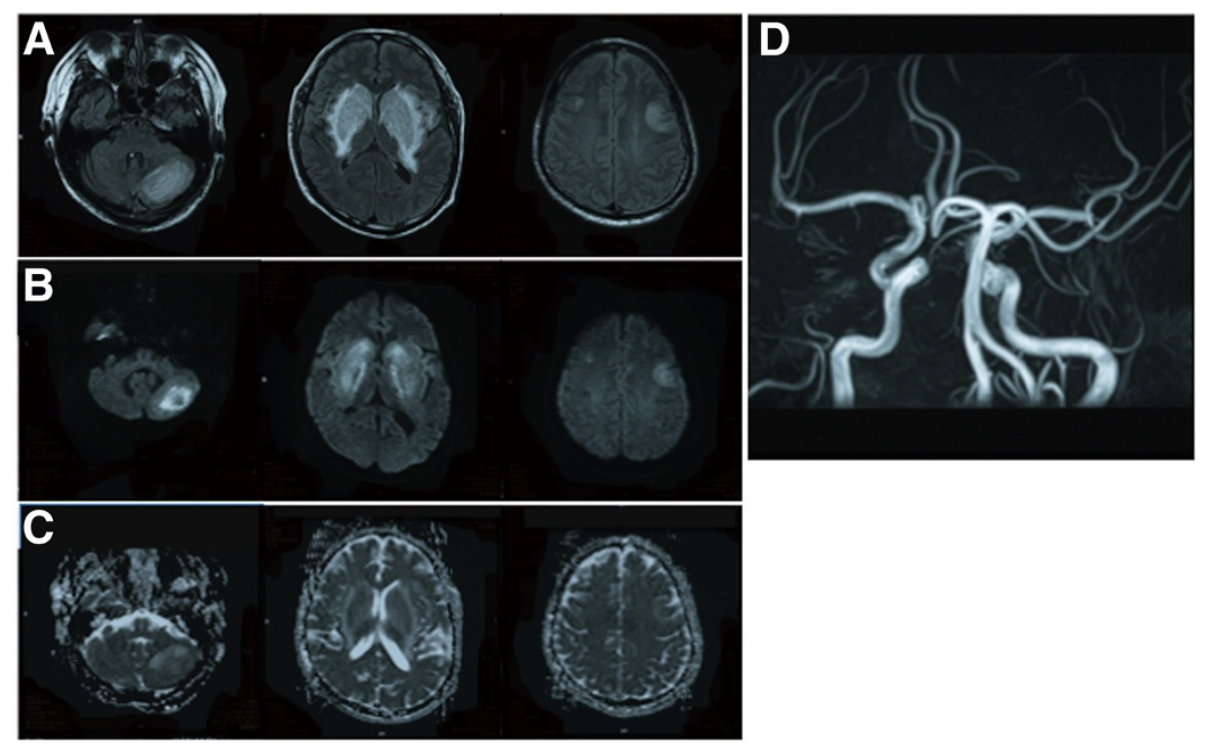

Figure 1 MR images on admission. Representative images of brain MRI including fluid attenuated inversion recovery (FLAIR) (A), diffusionweighted image (DWI) (B), apparent diffusion coefficient (ADC) map (C), and MR angiography (D) on admission, showing high-signal-intensity lesions in the bilateral basal ganglia, left cerebellar hemisphere, and bilateral frontal cortices. 


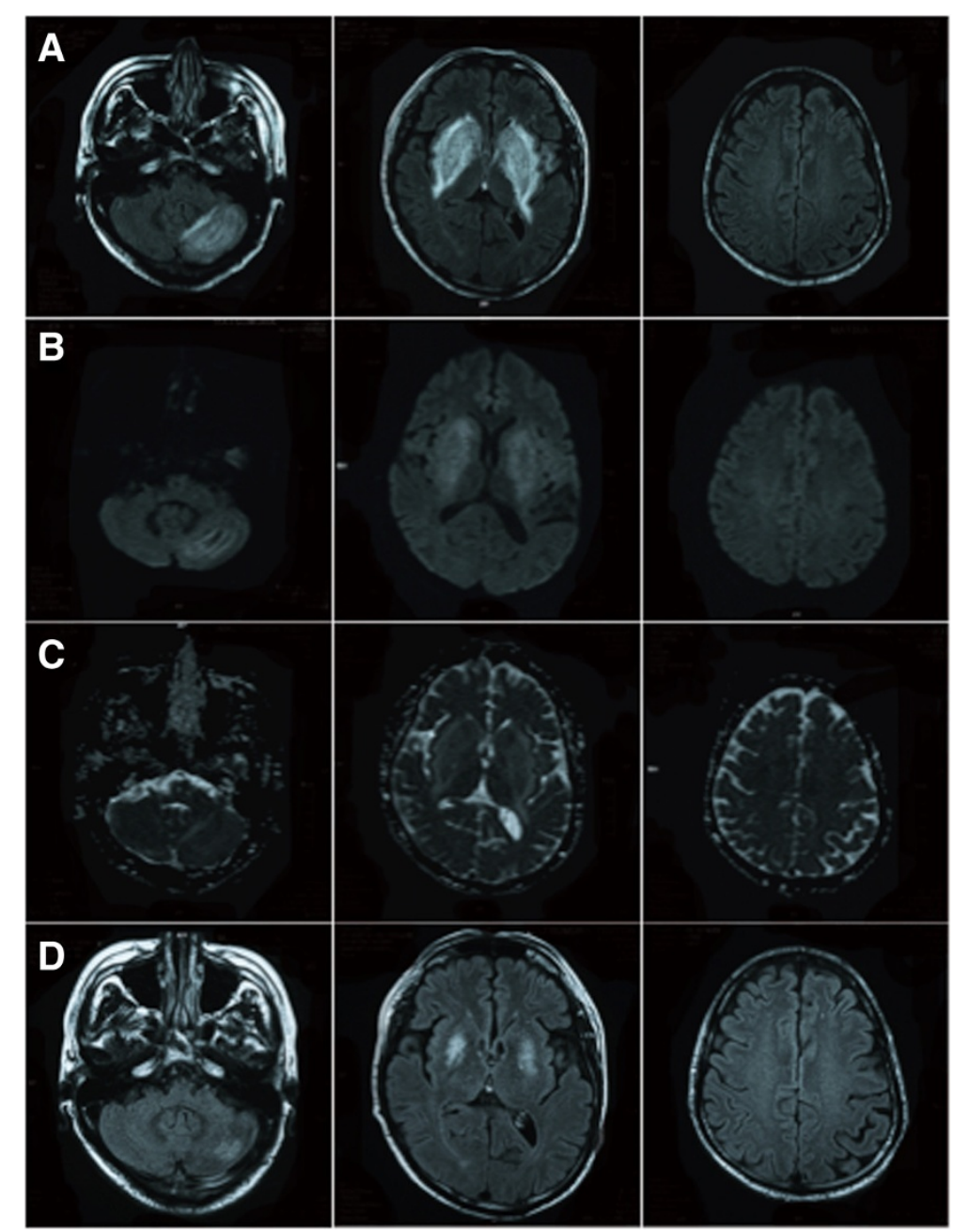

Figure 2 MR images at 1 and 4 weeks after admission. Representative FLAIR images (A), DWI (B), and ADC map (C) at 1 week after admission, and FLAIR images (D) at 4 weeks after admission.

FOG and postural instability are thought to be manifestations of dysfunction of pedunculopontine nucleus (PPN) and mesencephalic locomotor region (MLR), which are terminals with gamma-aminobutyric acidergic (GABAergic) output that are regulated by the globus pallidus internus (GPi) and substantia nigra pars reticulata $(\mathrm{SNr})$ [8]. Previously, abnormal signal intensity in GP, putamen, dorsolateral SN, and PPN on MRI were shown in patients with neoplasm, cerebrovascular disease, and CO intoxication who developed FOG [9-11]. Thus, disorder of GP, putamen, dorsolateral SN, and PPN can cause FOG. Although the precise pathophysiology underlying FOG remains unclear, we suggest that extensive vasogenic edema in the bilateral striatum reduced inhibition of $\mathrm{GPi} / \mathrm{SNr}$, and led secondarily to activation of the $\mathrm{GPi} / \mathrm{SNr}$ and suppression of the PPN/ MLR, thereby resulting in FOG and postural instability in the present case.
For patients with hypertension and undergoing hemodialysis, who suddenly develop FOG, physicians should be aware of the possibility of bilateral basal ganglionic lesions and PRES.

\section{Conclusions}

It is suggested that vasogenic edema on bilateral basal ganglia associated with PRES can cause acute transient FOG.

\section{Patient consent}

Written informed consent was obtained from the patient for publication of this case report and any accompanying images. A copy of the written consent is available for review by the Editor-in-Chief of this journal. 


\section{Competing interest}

The authors declare that they have no competing interests

\section{Authors' contributions}

Acquisition of data: AN and YU. Analysis and interpretation of data: AN, YU, HS, TK, and KN. Drafting of the manuscript: AN, YU, HS, and TU. Critical revision of the manuscript for important intellectual content: $Y U, N H$, and TU. All authors read and approved the final manuscript.

\section{Author details}

'Department of Neurology, Juntendo University Urayasu Hospital, 2-1-1 Tomioka, Urayasu, Chiba 279-0021, Japan. ²Department of Neurology, Juntendo University School of Medicine, 2-1-1 Hongo, Bunkyo, Tokyo

113-8421, Japan.

Received: 2 February 2013 Accepted: 28 June 2013

Published: 9 July 2013

\section{References}

1. Hinchey J, Chaves C, Appignani B, Breen J, Pao L, Wang A, Pessin MS, Lamy C, Mas JL, Caplan LR: A reversible posterior leukoencephalopathy syndrome. N Engl J Med 1996, 334:494-500.

2. Fugate JE, Claassen DO, Cloft HJ, Kallmes DF, Kozak OS, Rabinstein AA: Posterior reversible encephalopathy syndrome: associated clinical and radiologic findings. Mayo Clin Proc 2010, 85:427-432.

3. Giladi N: Freezing of gait. Clinical overview. Adv Neurol 2001, 87:191-197.

4. Factor SA: The clinical spectrum of freezing of gait in atypical parkinsonism. Mov Disord 2008, 23(Suppl 2):S431-S438.

5. Nutt JG, Bloem BR, Giladi N, Hallett M, Horak FB, Nieuwboer A: Freezing of gait: moving forward on a mysterious clinical phenomenon. Lancet Neurol 2011, 10:734-744.

6. Feekes JA, Cassell MD: The vascular supply of the functional compartments of the human striatum. Brain 2006, 129:2189-2201.

7. Takagi M, Wada H, Mukai K, Kihira H, Yano S, Minamikawa K, Wakita Y, Nakase T, Nagaya S, Deguchi K: Increased vascular endothelial cell markers in patients with chronic renal failure on maintenance haemodialysis. Blood Coagul Fibrinolysis 1994, 5:713-717.

8. Alexander GE, DeLong MR, Strick PL: Parallel organization of functionally segregated circuits linking basal ganglia and cortex. Annu Rev Neurosci 1986, 9:357-381.

9. Lee MS, Lyoo CH, Choi YH: Primary progressive freezing gait in a patient with CO-induced parkinsonism. Mov Disord 2010, 25:1513-1515.

10. Kuo SH, Kenney C, Jankovic J: Bilateral pedunculopontine nuclei stroke presenting as freezing of gait. Mov Disord 2008, 23:616-619.

11. Ishihara S, Kano O, Ikeda K, Shimokawa R, Kawabe K, Iwasaki Y: Clinicoradiological changes of brain NK/T cell lymphoma manifesting pure akinesia: a case report. BMC Neurol 2011, 11:137.

doi:10.1186/1471-2377-13-79

Cite this article as: Nakajima et al: Acute transient freezing of gait in a patient with posterior reversible encephalopathy syndrome. BMC Neurology 2013 13:79.

\section{Submit your next manuscript to BioMed Central and take full advantage of:}

- Convenient online submission

- Thorough peer review

- No space constraints or color figure charges

- Immediate publication on acceptance

- Inclusion in PubMed, CAS, Scopus and Google Scholar

- Research which is freely available for redistribution

Submit your manuscript at www.biomedcentral.com/submit 